\title{
Effectiveness of EEG Biofeedback as Compared with Methylphenidate in the Treatment of Attention-Deficit/Hyperactivity Disorder: A Clinical Outcome Study
}

\author{
Mohammad Ali Nazari ${ }^{1,2}$, Laurent Querne², Alain De Broca ${ }^{2}$, Patrick Berquin ${ }^{2}$ \\ ${ }^{1}$ Department of Psychology, University of Tabriz, Tabriz, Iran; ${ }^{2}$ Department of Paediatric Neurology, Lab. Neurosciences Fonction- \\ nelles \& Pathologies, Amiens, France. \\ Email: alinazari@tabrizu.ac.ir
}

Received March 13 ${ }^{\text {th }}$, 2010; revised March 23 ${ }^{\text {rd }}$, 2011; accepted April 25 ${ }^{\text {th }}, 2011$.

\begin{abstract}
Operant conditioning of the electroencephalographic rhythm (EEG biofeedback) is argued to be an effective method for treating children with ADHD. This study was designed to evaluate whether this method, compared to methylphenidate, achieves an equally effective outcome. Participants were 39 children aged between $7-12$ years. Thirteen children with attention-deficit/hyperactivity disorder (ADHD) were trained to enhance the amplitude of the beta1 activity $(15-18 \mathrm{~Hz})$ and decrease the amplitude of the theta activity $(4-8 \mathrm{~Hz})$, and 13 of which were treated with methylphenidate alone. Thirteen healthy children did not receive intervention. Several behavioral, neuropsychological and experimental tests were administered before and after intervention. While behavioral measures were improved by both types of method, methylphenidate was significantly more effective than EEG biofeedback. Response inhibition was improved only by EEG biofeedback. Both EEG biofeedback and methylphenidate were associated with improvements on the variability and accuracy measures of computerized tests. Intellectual ability increased also by both methods. Although averaged effect size for methylphenidate seems to be greater than for EEG biofeedback, the difference was not significant. In conjunction with other studies, these findings demonstrate that EEG biofeedback can significantly improve several behavioral and cognitive functions in children with $A D H D$, and it might be an alternative treatment for non-responders or incomplete responders to medication as well as for those their parents favor a non-pharmacological treatment.
\end{abstract}

Keywords: ADHD, Electroencephalography, EEG biofeedback, Neurofeedback, Methylphenidate

\section{Introduction}

Attention-deficit/hyperactivity disorder (ADHD) requires ongoing management and monitoring. Although robust evidence indicates the efficacy of stimulant medications in helping to manage the symptoms of school-aged ADHD children [1], typically improvements are noted in some functional domains but not in others [2]. Furthermore, only $60 \%$ to $75 \%$ of DSM-IV—-diagnosed ADHD subjects respond to methylphenidate [3-9]. As concluded by Pelham [10], "other interventions are needed for non-responders or incomplete responders to medication".

Neurofeedback, which has also been called electroencephalography (EEG) biofeedback, is reportedly used by more than 1500 practitioners [11]. The theoretical basis of neurofeedback is based on a biological model of
ADHD, considering that ADHD is a disorder of neural regulation and assuming under this approach that these neural deficiencies are amenable to change using behavioral methods (e.g. an operant conditioning procedure whereby an individual learns to self-regulate the electrical activity) [11]. Since the work of Lubar and Shouse [12], several studies have used neurofeedback approaches for treating ADHD and concluded that despite some limitations, neurofeedback may be worthy of further consideration as a viable treatment approach for ADHD [13-28]; for a meta-analysis see Arns et al. [29]. However, despite these promising results, neurofeedback has not been considered a standard therapy for ADHD [28] and has been considered an inconsistent and problematic method [30]. More experimental investigations need to 
evaluate the cognitive and behavioral outcome following neurofeedback [31,32].

The main issue of interest was whether neurofeedback achieves an equally effective outcome as compared with stimulant medication (as a standard method). In addition, we investigated whether the two types of treatment had dissimilar effects on different domains of behavioral and cognitive functioning of children with ADHD as measured by clinical, neuropsychological and experimental tests.

\section{Methodology}

\subsection{Design}

Considering the ethical problem of including untreated patient or patient undergoing placebo, La Vaque and Rossiter [33] pointed out that, rather than comparing a new treatment (e.g., neurofeedback) to a no-treatment placebo, it should be compared to a protocol of "known efficacy" to determine whether such an intervention would result in an equivalent effect. This type of design is often referred to equivalent study [34]. Regarding the well established efficacy of methylphenidate [1], we compared the effects of neurofeedback and methylphenidate. A control group made up of healthy children to control test-retest effect.

\subsection{Participants and Selection Procedure}

The ADHD children were all recruited from the pediatric neurology department of Amiens University Hospital. These children had never been treated with methylphenidate. Diagnosis was based on Diagnostic and Statistical Manual of Mental Disorders, Fourth Edition (DSM-IV) criteria and inclusion was dependent on meeting the full diagnostic criteria for ADHD. For all participants, the Child Behavior Checklist (CBCL) [35] was completed by parents, and the SNAP-IV questionnaire [36] was filled out by parents and teachers. Diagnosis was then established after semi-structured interview, a clinical neurological examination, and a set of ADHD-oriented neuropsychological and behavioral tests including the ADHD Rating Scale-IV [37] full version of the WISC-III [38], the Stroop Color-Word test $[39,40]$. Then they were administered the Continuous Performance Test (CPT-II) [41] and an attentional capture test (ACT) $[42,43]$. These cases were reviewed independently by a pediatric neurologist and a psychologist blinded to each other's findings and included in the ADHD group only if both clinicians concurred on this diagnosis. This protocol was approved by the local ethics committee. No monetary compensation was given.

Participants were selected according to the following criteria: age between 7 and 12 years; performance and verbal-scales of IQ > 80; and no additional neurologic disorders.

Originally, 40 children with ADHD participated in the study; afterwards 12 children were excluded as follows: 11 children had performance and/or verbal-scale IQ $<80$; and one child had burst of diffuse irregular slow wave activities in her EEG. Hence, 28 children with ADHD participated in two treatment groups: neurofeedback (NFT) $(n=13)$ or methylphenidate (MPH) $(n=15)$ group. Assignment to the treatment groups was based on the parents' informed choice. NFT participants did not receive any psychoactive medication during the entire study period. All participants of the NFT group completed the treatment schedule, whereas two participants of the MPH group dropped out; one of them because of excessive side effects and another one did not like to continue to take methylphenidate. This left a total of 26 children with ADHD for analysis.

As mentioned, 13 age-matched healthy children participated as the no-treating control group (CON). Therefore, our study population consisted of 39 children as follows:

- 13 ADHD children $(12$ males, mean age $=(9.1 \pm 1)$ SD years) as NFT group;

- 13 ADHD children (all male, mean age $=(8.8 \pm 1.8)$ SD years) as MPH group;

- 13 healthy children $(10$ males, mean age $=(9.1 \pm 1.3)$ $\mathrm{SD}$ years) as $\mathrm{CON}$ group.

\subsection{Pre- and Post-Test Measures}

Following measures were used for statistical propose:

- Behavioral test: the SNAP-IV which were filled out by parents and teachers. The SNAP-IV is a rating scale based on symptoms listed in the DSM-IV and includes the two subsets of symptoms: inattention and hyperactivity/impulsivity.

- Neuropsychological tests: full version of the WISC-III was performed to assess the intellectual ability. The Stroop was used to evaluate the interference score, which represents response inhibition $[44,45]$.

- Experimental tests: the CPT-II and the ACT. The CPT is a task evaluating capacity to maintain attention during time and capacities to inhibit a prepotent motor response $[41,46]$. Four main scores of the CPT-II were used: reaction time (RT), standard error of RT as a variability, omission, and commission errors. The ACT is another objective test based on attentional capture paradigm. The dependent measures in this task were RT, variability, and error response.

For the NFT and MPH groups, all instruments were 
performed after treatment, whereas healthy children (CON) were reassessed via only the CPT-II and the ACT. All MPH participants were "wash-on" when post-test was administered.

\subsection{Neurofeedback Training}

Training was administered using the ProComp2 ${ }^{\circledR}$ encoder (Thought Technology Ltd.) and the NeuroCARE Pro ${ }^{\circledR}$, version 1.9 (Zengar Institute Inc.). EEG was recorded from two electrodes placed on C3 and C4 according to the International 10 - 20 system [47], referenced to linked earlobes (sampling rate: $256 \mathrm{~Hz}$ ).

Training protocol was similar to the paradigm described by Lubar et al. [15]. The ongoing EEG with frequency range $1-60 \mathrm{~Hz}$ was band-pass filtered in two frequency ranges: theta $(4-8 \mathrm{~Hz})$ and beta1 $(15-18 \mathrm{~Hz})$. The aim of neurofeedback training was to increase the power in the beta1 band ("reward band") and simultaneously to decrease the power in the theta band ("inhibit band"). The trainer, seated behind participant, observed information about the power in each of these frequency bands online on a monitor, and fed back audio-visually to the participant via a second monitor. Feedback consisted of negative feedback and reinforcement: whenever spectral amplitudes were below or above thresholds for inhibit or reward bands, the software briefly interrupts ongoing movie.

Twenty four neurofeedback training sessions were conducted during 10 to 12 weeks with 2 - 3 sessions per week on afternoons, weekdays or vacations. Each session consisted of 35 - $40 \mathrm{~min}$, lasted $\sim 1$ hour including the time for preparation. Participants sat in a comfortable chair in front of the monitor on which the movie was shown. Our instructions were "let the movie play" and "be calm and attentive to make the movie play better". Children were asked to pay attention to history of the movie. They were explicated that the amount of pauses were not sign of improvement or regression. However, it was informed that the pauses in the movie were giving them information, as an indication of attention, and there was no other technical error.

\subsection{Methylphenidate Administration}

Participants of the MPH group were administered 20 $\mathrm{mg} /$ day (average dose $(0.71 \pm 0.13) \mathrm{mg} / \mathrm{kg}$ ) long action methylphenidate (Ritalin LP $^{\circledR}$ ) every day on morning during the entire treatment period.

\subsection{Statistical Analysis}

Conventional Tests: Analyses of variance (ANOVA) for repeated measurements with the factors Treatment as the within-subjects factors and Group as the between-sub- jects factor were separately calculated for each dependent measure. Effect sizes (ESs) were assessed with Cohen's $d$ [48].

Equivalence Analysis: for data reduction purpose and investigating the "equally effect of two types of treatment”, we performed the following steps:

- ESs of each dependent measure were calculated for each group, separately;

- Calculated effect sizes were averaged for NFT and MPH group, separately;

- To test the conventional "no difference" null hypothesis between mean ESs of two groups, independent $\mathrm{t}$ test was used.

- A statistically significant difference between two treatments may or may not be equivalent for practical purposes [49]. Equivalence analysis [50-51] was conducted to test whether the treatment-related changes in both groups could be regarded as statistically equivalent. Using equivalent analysis, we investigated "equally effect of two types of treatment" to determine whether possible differences between neurofeedback and methylphenidate were significant for clinical purposes.

\section{Results}

Means and standard deviations of the Pre-test and posttest of all dependent measures with results of paired $t$ tests and ESs for the NFT and MPH groups are presented in Table 1. The effects of both types of treatment on the each dependent measure, as indicated by the ESs, are compared in Figure 1.

\subsection{Behavioral Effect}

There were no significant differences between MPH and NFT groups on any of the SNAP-IV scales at pre-test. According to parent's opinion, inattention (F1, $24=36.55$; $P=0.000 ; \mathrm{ES}=0.60)$ and hyperactivity $(\mathrm{F} 1,24=41.09$; $P=0.000$; ES $=0.63$ ) were improved by both types of treatment. An interaction Treatment $\times$ Group effect was found for inattention (F1, $24=9.75 ; P=0.005$; $\mathrm{ES}=0.29$ ) and hyperactivity (F1, $24=7.82 ; P=0.01$; ES $=0.25$ ). As depicted in Table 1 and Figure 1, treatment effect was more pronounced in the MPH group.

\subsection{Neuropsychological Effect}

- Stroop Color-Word Test: seventeen children (9 in NFT and 8 in MPH group) were included for the analyses of the Stroop Test. Three children were younger than 8 years and six children had below $2 z-$ score in the word reading score.

At pre-test, the NFT group had more problems in interference control as compared with MPH group but did 
Table 1. Means and standard deviations of the Pre-test [M1 (SD)] and the post-test [M2 (SD)] of dependent measures with results of paired $t$ tests and effect sizes (ES) for the Neurofeedback and Methylphenidate groups.

\begin{tabular}{|c|c|c|c|c|c|c|c|c|c|c|}
\hline & \multicolumn{5}{|c|}{ Neurofeedback } & \multicolumn{5}{|c|}{ Methylphenidate } \\
\hline & M1 (SD) & M2 (SD) & $t$ test & $P$ & ES & M1 (SD) & M2 (SD) & $t$ test & $P$ & ES \\
\hline SNAP-IV_In & $2.06(0.51)$ & $1.75(0.55)$ & 2.39 & 0.03 & 0.58 & $2.4(0.29)$ & $1.43(0.6)$ & 5.79 & 0.000 & 2.05 \\
\hline SNAP-IV_Hyp & $1.8(0.73)$ & $1.37(0.8)$ & 3.19 & 0.008 & 0.56 & $2.4(0.45)$ & $1.3(0.79)$ & 5.58 & 0.000 & 1.7 \\
\hline Stroop & $-1.15(0.8)$ & $-0.19(0.6)$ & 5.84 & 0.000 & 1.35 & $-0.36(0.78)$ & $-0.38(1.2)$ & 0.04 & 0.96 & 0 \\
\hline IQ_Full & $97.8(9.2)$ & $103.9(13.2)$ & 2.81 & 0.01 & 0.53 & 88.5 (6.9) & $94.6(11.4)$ & 2.14 & 0.05 & 0.64 \\
\hline IQ_Per & $95.1(13.3)$ & $104.2(16.2)$ & 3.8 & 0.003 & 0.61 & 83.9 (6.7) & $96(14.6)$ & 3.18 & 0.005 & 1.06 \\
\hline IQ_Verb & 101.5 (12.5) & $103.4(14.9)$ & 0.81 & 0.43 & 0.13 & $94.6(7.9)$ & $94.1(8.8)$ & 0.24 & 0.81 & 0.05 \\
\hline CPT-II_RT & $55.9(11.3)$ & $54.9(10.1)$ & 0.43 & 0.67 & 0.09 & $56.1(11.5)$ & $53.7(10.2)$ & 1.21 & 0.24 & 0.22 \\
\hline CPT-II_Var & $58.2(9.7)$ & $52.9(10.6)$ & 2.28 & 0.04 & 0.52 & $57(9.2)$ & 49.3 (8.3) & 3.27 & 0.007 & 0.87 \\
\hline CPT-II_Om & $57.8(11.8)$ & 48.7 (6.2) & 4.46 & 0.001 & 0.96 & $58.9(14.4)$ & $47(5.3)$ & 3.67 & 0.003 & 1.09 \\
\hline ACT_RT & 515 (113) & 478 (98) & 1.21 & 0.24 & 0.34 & 526 (155) & 450 (98) & 2.9 & 0.01 & 0.58 \\
\hline ACT_Var & $192(62)$ & 154 (49) & 2.19 & 0.04 & 0.68 & 179 (77) & $120(44)$ & 3.22 & 0.007 & 0.94 \\
\hline ACT_Error & 11.4 (9.3) & $8.3(8.3)$ & 0.82 & 0.42 & 0.35 & $7.5(9)$ & $5(6.7)$ & 0.8 & 0.43 & 0.31 \\
\hline
\end{tabular}

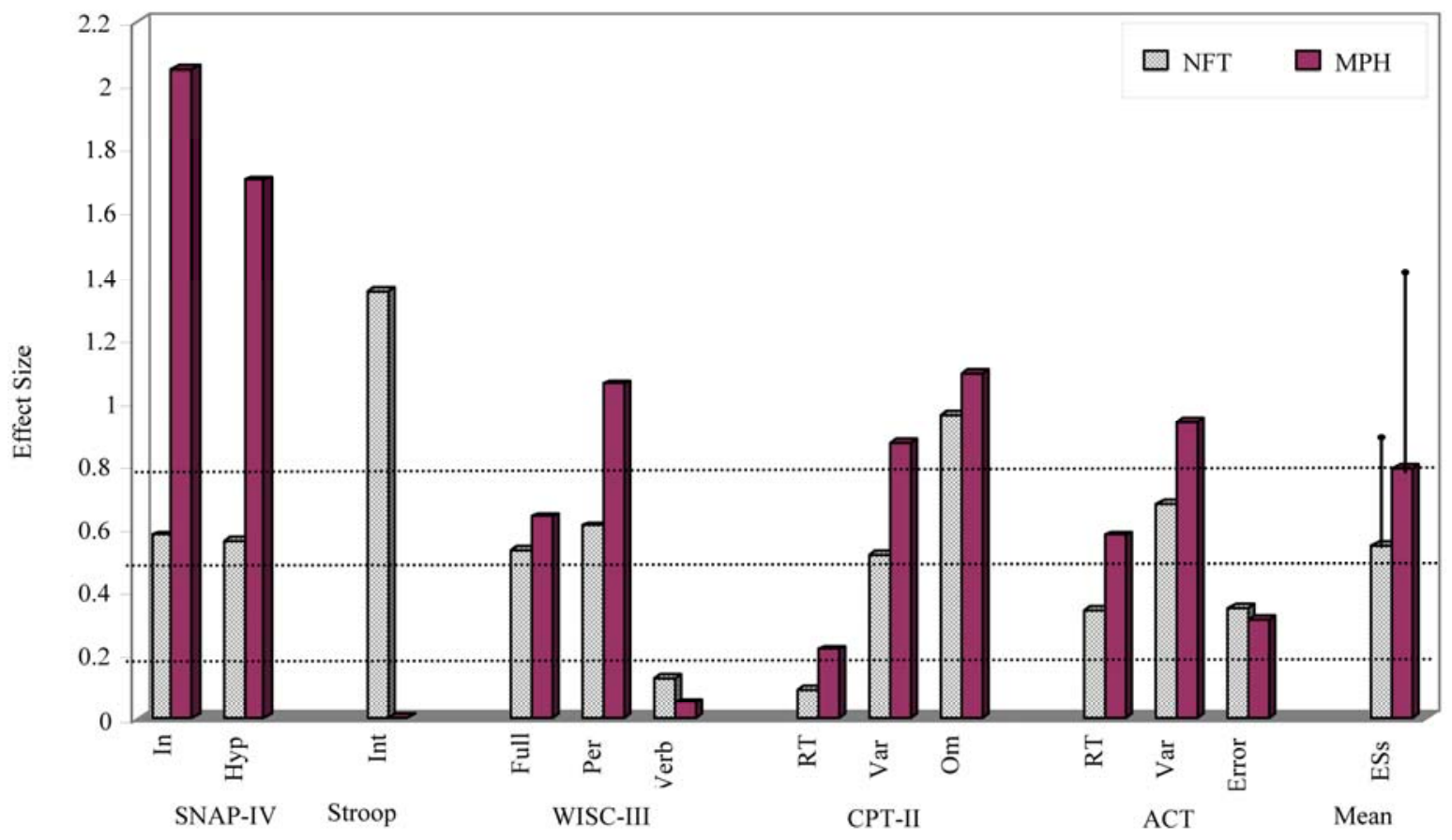

Figure 1. Effect sizes of each dependent measures and mean (+1 SD) of these effect sizes for NFT and MPH groups. Note: ACT = Attentional Capture test; ESs = mean effect sizes of all dependent measures; Hyp = Hyperactivity; In = Inattention; Int $=$ Interference.

not reach significant $(P=0.06)$. There were a significant main effect of Treatment $(\mathrm{F} 1,15=7.14 ; P=0.01$; $\mathrm{ES}=$ $0.32)$ and Treatment $\times$ Group interaction $(F 1,15=7.6 ; P$ $=0.01$; ES $=0.33$ ). Paired $t$ test revealed that the interference score was significantly improved by only NFT.

- WISC-III: there were no significant differences between groups on any of the WISC-III scores at pre- test. Main effects of Treatment were identified for the full IQ scale (F1, $24=11.65 ; P=0.002$; ES = 0.38). Analysis of the subscores demonstrated that this effect was accounted for mainly by the performance score (F1, $24=22.24 ; P=0.000 ; \mathrm{ES}=0.48)$, but not by the verbal score $(\mathrm{F} 1,24=0.17 ; P=0.67$; ES = 0.007). Further analysis confirmed that all subtests of 
the performance score, except Block Design, increased significantly from pre- to post-test. Main effect of Treatment was significant for Picture Completion (F1, 24 = 13.74; $P=0.001 ; \mathrm{ES}=0.36$ ), Coding (F1, $24=$ 41.09; $P=0.000$; ES $=0.63$ ), Picture Arrangement (F1, $24=4.84 ; P=0.03$; ES $=0.17$ ), and Object Assembly (F1, $24=10.25 ; P=0.004$; ES $=0.29$ ) for MPH group only. Regarding the verbal subtests, except Digit Span (F1, $24=10.96 ; P=0.003$; ES = 0.31 ), none of the subtests were significantly changed. There were no further significant main effects or interactions for any of the three scores or subtests.

\subsection{Experimental Effect}

- CPT-II: there were pre-test differences between groups on the omission errors (F2, $36=5.74 ; P=$ $0.007)$ and variability of RT (F2, $36=4.59 ; P=0.01)$. Post hoc LST test yielded significant between ADHD (NFT and MPH groups) and control children $(P<$ 0.008 for omission errors and $P<0.01$ for variability), but not between NFT and MPH groups. No significant differences between groups (NTF versus MPH versus $\mathrm{CON}$ ) were found for the commission errors RT at pre-test.

For omission errors, a significant main effect of Treatment (F1, $36=17.46 ; P=0.000$; ES $=0.32)$ and Treatment $\times$ Group interaction (F2, $36=9.24 ; P=0.001$; ES = 0.33 ) was identified. Here, improvements were observed from pre- to post-test in both NFT and MPH groups, but not in control children. Three groups did not differ significantly at post-test. Similarly, there were a significant main effect of Treatment $(\mathrm{F} 1,36=6.43 ; P=0.01$; $\mathrm{ES}=$ $0.15)$ and Treatment $\times$ Group interaction (F2, $36=4.06$; $P=0.02$; ES $=0.18$ ) for the variability which improved by both treatments. Changes in control children were not significant. Three groups did not differ significantly at post-test. Although main effect of Treatment was significant (F1, $36=17.74 ; P=0.000$; ES $=0.33$ ) for commission errors, there was no significant Treatment $x$ Group interaction. Commission errors were significantly reduced from pre- to post-test in control children as well $(t 12=2.36 ; p=0.03 ; \mathrm{ES}=0.51)$. Furthermore, since this measure was not statistically different between the ADHD and control groups it could not be a viable measure in this study, hence was excluded from further analysis. For RT, there was no significant main effect (Treatment, Group) or interaction.

- Attentional Capture Test: for RT, no significant differences between groups (NTF versus MPH versus $\mathrm{CON}$ ) were found at pre-test. However, we observed a significant main effect of Treatment (F1, $36=5.43$; $P=0.02 ; \mathrm{ES}=0.13$ ), but no significant Treatment $\times$
Group interaction. Paired t test revealed that response speed was significantly improved by only methylpenidate. Changes in NFT group and control children were not significant. Three groups did not differ significantly at post-test. Although the effect of distractors was significant on RT for distractor trails (507 \pm 35) compared to control trails ( $493 \pm 130$; $t 38=2.36$; $P=0.02$ ), there was no significant main effect (Treatment, Group) or interaction.

Pre-test differences between groups on the variability of RT (F2, $36=6.04 ; P=0.005$ ) were found. These differences were between ADHD and controls $(P<0.01)$, but not between NFT and MPH groups. Main effect of Treatment $(\mathrm{F} 1,36=12.07 ; P=0.001 ; \mathrm{ES}=0.25)$ was significant. Effect of Treatment $\times$ Group interaction was marginally significant $(\mathrm{F} 2,36=3.17 ; P=0.054 ; \mathrm{ES}=$ 0.15 ). While changes in control children were not significant, variability was reduced by both treatments. Three groups did not differ significantly at post-test.

At pre-test, control children had less response error, but was not significant. Significant main effect (Treatment, Group) or interaction was not also observed.

\subsection{All Dependent Variables}

As shown in Table 2 and Figure 1, while ESs by MPH $(\mathrm{M}=0.791, \mathrm{SD}=0.631)$ is greater than by NFT $(\mathrm{M}=$ $0.558, \mathrm{SD}=0.344)$ treatment, this difference was not significant. The equivalent analysis indicated that NFT and MPH did not also equivalent.

\section{Discussion}

Based on the SNAP-IV, parents reported reduced ADHDrelated behaviors in both groups; however, MPH was more effective than NFT. Although two groups were not significantly different at pre-test, larger MPH effect might obtain from degraded inattention and hyperactivity in the participants of MPH group.

In terms of response inhibition, experimental data demonstrated that the effect of MPH on the interference score of the Stroop test is inconclusive [52]. While some studies $[42,53]$ have not found significant effect, some others $[52,54,55]$ have reported a positive effect. To our knowledge, no study has compared NFT with MPH effect by using the Stroop test. Nevertheless, in an fMRI study using Counting Stroop test, not only increased accuracy in interference trails, but also activation in the right anterior cingulate cortex was observed in NFT compared with no-treatment ADHD group [26]. In the present study, positive treatment effect was found only for NFT. One explanation could be that NFT group benefit more than MPH group because of degraded interference control in pre-treatment. Future research is 
Table 2. Conventional and equivalence test results of the means and standard deviations of the effect size of all dependent measures for the neurofeedback (NFT) and methylphenidate (MPH) groups.

\begin{tabular}{|c|c|c|c|c|c|c|c|c|c|}
\hline \multicolumn{2}{|c|}{ NFT } & \multicolumn{2}{|c|}{ NFT } & \multicolumn{3}{|c|}{ Conventional Test } & \multicolumn{3}{|c|}{ Equivalence Test ${ }^{1}$} \\
\hline M & SD & M & SD & $t$ test & $d f$ & $P$ & Criterion $^{2}$ & $t$ test 1 & $t$ test 1 \\
\hline 0.558 & 0.344 & 0.791 & 0.631 & 1.12 & 22 & 0.27 & 0.078 & 1.50 & 0.47 \\
\hline
\end{tabular}

${ }^{1}$ One-tailed critical $t$-value (for $P<0.05$ ) $=1.72$; ${ }^{2}$ Criterion is $\pm 10 \%$ of the MPH group mean.

recommended to investigate the effect of NFT and MPH on interference control, as a form of response inhibition.

In accordance with other studies $[15,17,18,21,28]$, intellectual ability as assessed with the WISC-III improved by both NFT and MPH. It is likely that the observed changes were attributable to practice effects [21]. However, the improvement on the intelligence performance scores, but not on the verbal one, allow us to suggest that this improvement is related to attention enhancement. In this line, improvement on the digit span subtest, which is related to the attention/executive function [56] seems to confirm our conclusion.

Cognitive improvement by both types of treatment was also observed using two experimental tests, confirming that improvements were not limited to parent's opinion. Treatment effect size was larger in the RT-variability and omissions, especially in the CPT-II. These behavioral, neuropsychological and experimental findings accord with those of previous studies demonstrating that NFT can significantly improve several behavioral and cognitive functions in ADHD children [12-28].

Calculated NFT and MPH effect sizes for each dependent measure were different. It would suggest that the impact of the two types of treatment on different domains of behavioral and cognitive functioning of children with ADHD did not similar.

With respect to the performed calculations on all measures, we found that although averaged effect size for MPH was greater than for NFT, both were in medium range and the difference was not significant. The equivalent analysis indicated that NFT and MPH did not also equivalent. These results (not different obtained from conventional tests, but not equivalent obtained from equivalence analysis) demonstrate that the variability is too great relative to the effect size to interpret. The small sample size limits the interpretability of the clinical outcome comparison. Proving the equivalence of both treatments would require a much larger sample.

The present study overcame some aforementioned methodological shortcoming [30] by comparing NFT with a standard method (MPH) and by applying both objective assessments and external ratings by parents. Nevertheless, our study had a number of limitations. Lack of random assignment may result in possible dif- ferences in treatment motivation and effects of expectancies. However, it was not feasible to administer either treatment without the parents' consent. Although it is tempting to conclude that specific training in changing brainwave activity was responsible for the treatment effects, this conclusion cannot be made with certainty. The study design did not enable this possibility to conclusively rule out because of ethical issues.

Overall, our findings might provide further support to the view that neurofeedback can be considered an effective treatment for children with ADHD, at least an appropriate adjunctive treatment for non-responders or incomplete responders to medication as well as for those their parents favor a non-pharmacological treatment.

\section{Acknowledgements}

We would like to acknowledge all parents of participants and Mme Pataille for the recruitment of the control children. We gratefully thank Dr John Vessy, Wheaton College, for providing advice and feedback regarding the equivalence analysis.

\section{REFERENCES}

[1] R. T. Brown, R. W. Amler, W. S. Freeman, J. M. Perrin, M. T. Stein, H. M. Feldman, et al., "Treatment of Attention-Deficit/Hyperactivity Disorder: Overview of the Evidence,” Pediatrics, Vol. 115, No. 6, 2005, pp. 749-757. doi:10.1542/peds.2004-2560

[2] W. J. Pelham and B. H. Smith, "Prediction and Measurement of Individual Responses to Ritalin by Children and Adolescents with ADHD,” In: L. Greenhill and B. Osman, Eds., Ritalin: Theory and Patient Management, 2nd Edition, Mary Ann Liebert Inc., New York, 2000.

[3] J. Elia, "Drug Treatment for Hyperactive Children: Therapeutic Guidelines,” Drugs, Vol. 46, No. 5, 1993, pp. 863-871.

[4] J. K. Nash, “Treatment of Attention Deficit Hyperactivity Disorder with Neurotherapy," Clinical Electroencephalography, Vol. 31, No. 1, 2000, pp. 30-37.

[5] T. Spencer, J. Biederman and T. Wilens, "Pharmacotherapy of Attention Deficit Hyperactivity Disorder," Child and Adolescent Psychiatry Clinics of North America, Vol. 9, No. 1, 2000, pp. 77-97.

[6] D. A. Waschbusch and G. P. Hill, "Empirically Supported, Promising, and Unsupported Treatments for Children 
with Attention-Deficit/Hyperactivity Disorder,” In: S. O. Lilienfield, S. J. Lynn and J. M. Lohr, Eds., Science and Pseudoscience in Clinical Psychology, Guilford Press, New York, 2003.

[7] MTA Cooperative Group, "National Institute of Mental Health Multimodal Treatment Study of ADHD Follow-up: Changes in Effectiveness and Growth after the End of Treatment,” Pediatrics, Vol. 113, No. 4, 2004, pp. 762769.

[8] J. Biederman and S. V. Faraone, "Attention-Deficit Hyperactivity Disorder,” Lancet, Vol. 366, No. 9481, 2005, pp. 237-248.

[9] L. C. Smoot, L. A. Boothby and R. C. Gillett, "Clinical Assessment and Treatment of ADHD in Children," International Journal of Clinical Practice, 2007, Vol. 61, No. 10, pp. 1730-1738.

[10] W. E. Pelham, "Psychosocial Interventions for ADHD," In: P. S. Jensen and J. R. Cooper, Eds., Attention Deficit Hyperactivity Disorder: State of the Science: Best Practices, Civic Research Institute, New Jersey, 2002, pp. 12-13.

[11] S. M. Butnik, "Neurofeedback in Adolescents and Adults with Attention Deficit Hyperactivity Disorder," Journal of Clinical Psychology, Vol. 61, No. 5, 2005, pp. 621-625. doi:10.1002/jclp.20124

[12] J. F. Lubar and M. N. Shouse, "EEG and Behavioral Changes in a Hyperkinetic Child Concurrent with Training of the Sensorimotor Rhythm (SMR): A Preliminary Report,” Biofeedback Self Regulation, Vol. 1, No. 3, 1976, pp. 293-306. doi:10.1007/BF01001170

[13] M. N. Shouse and J. F. Lubar, "Operant Conditioning of EEG Rhythms and Ritalin in the Treatment of Hyperkinesis,” Biofeedback Self Regulation, Vol. 4, No. 4, 1979, pp. 299-312. doi:10.1007/BF00998960

[14] J. O. Lubar and J. F. Lubar, "Electroencephalo-Graphic Biofeedback of SMR and Beta for Treatment of Attention Deficit Disorders in a Clinical Setting," Biofeedback Self Regulation, Vol. 9, No. 1, 1984, pp. 1-23. doi:10.1007/BF00998842

[15] J. F. Lubar, M. O. Swartwood, J. N. Swartwood and P. H. O’Donnell, “ Evaluation of the Effectiveness of EEG Neurofeedback Training for ADHD in a Clinical Setting as Measured by Changes in T.O.V.A. Scores, Behavioral Ratings, and WISC-R Performance," Biofeedback Self Regulation, Vol. 20, No. 1, 1995, pp. 83-99. doi:10.1007/BF01712768

[16] T. R. Rossiter and T. J. La Vaque, "A Comparison of EEG Biofeedback and Psycho Stimulants in Treating Attention Deficit/Hyperactivity Disorders," Journal of Neurotherapy, Vol. 1, 1995, pp. 48-59. doi:10.1300/J184v01n01_07

[17] M. Linden, T. Habib and V. Radojevic, "A Controlled Study of the Effects of EEG Biofeedback on Cognition and Behavior of Children with Attention Deficit Disorder and Learning Disabilities,” Biofeedback Self Regulation, Vol. 21, No. 1, 1996, pp. 35-49.

\section{doi:10.1007/BF02214148}

[18] L. Thompson and M. Thompson, "Neurofeedback Combined with Training in Metacognitive Strategies: Effectiveness in Students with ADD," Applied Psychophysiology and Biofeedback, Vol. 23, No. 4, 1998, pp. 243-263. doi:10.1023/A:1022213731956

[19] D. P. Carmody, D. C Radvanski, S. Wadhwani, M. J, Sabo and L. Vergara, "EEG Biofeedback Training and Attention-Deficit/Hyperactivity Disorder in an Elementary School Setting,” Journal of Neurotherapy, Vol. 4, 2001, pp. 5-27.

[20] V. J. Monastra, D. M, Monastra and S. George, "The Effects of Stimulant Therapy, EEG Biofeedback, and Parenting Style on the Primary Symptoms of Attention-Deficit/Hyperactivity Disorder,” Applied Psychophysiology and Biofeedback, Vol. 27, No. 4, 2002, pp. 231-249. doi:10.1023/A:1021018700609

[21] T. Fuchs, N, Birbaumer, W. Lutzenberger, J. H. Gruzelier and J. Kaiser, "Neurofeedback Treatment for Attention-Deficit/Hyperactivity Disorder in Children: A Comparison with Methylphenidate," Applied Psychophysiology and Biofeedback, Vol. 28, No. 1, 2003, pp. 1-12. doi:10.1023/A:1022353731579

[22] C. Heywood and I. Beale, "EEG Biofeedback vs Placebo Treatment for Attention-Deficit/Hyperactivity Disorder: A Pilot Study,” Journal of Attentional Disordered, Vol. 7, No. 1, 2003, pp .43-55.

[23] B. H. Cho, S. Kim, D. I. Shin, J. H. Lee, S. M. Lee, I. Y. Kim, et al., "Neurofeedback Training with Virtual Reality for Inattention and Impulsiveness," Cyberpsychology Behavior, Vol. 7, No. 5, 2004, pp. 519-526.

[24] H. Heinrich, H. Gevensleben, F. J. Freisleder, G. H. Moll and A. Rothenberger, "Training of Slow Cortical Potentials in Attention-Deficit/Hyperactivity Disorder: Evidence for Positive Behavioral and Neurophysiological Effects,” Biological Psychiatry, Vol. 55, No. 7, 2004, pp. 772-775. doi:10.1016/j.biopsych.2003.11.013

[25] T. Rossiter, "The Effectiveness of Neurofeedback and Stimulant Drugs in Treating AD/HD: Part II. Replication," Applied Psychophysiology and Biofeedback, Vol. 29, No. 4, 2004, pp. 233-243. doi:10.1007/s10484-004-0383-4

[26] M. Beauregard and J. Levesque, "Functional Magnetic Resonance Imaging Investigation of the Effects of NeuroFeedback Training on the Neural Bases of Selective Attention and Response Inhibition in Children with Attention-Deficit/Hyperactivity Disorder," Applied Psychophysiology and Biofeedback, Vol. 31, No. 1, 2006, pp. 3-20. doi:10.1007/s10484-006-9001-y

[27] J. Levesque, M. Beauregard and B. Mensour, "Effect of Neurofeedback Training on the Neural Substrates of Selective Attention in Children with Attention-Deficit/ Hyperactivity Disorder: A Functional Magnetic Resonance Imaging Study,” Neuroscience Letter, Vol. 394, No. 3, 2006, pp. 216-221. doi:10.1016/j.neulet.2005.10.100

[28] U. Strehl, U. Leins, G. Goth, C. Klinger, T. Hinterberger 
and N. Birbaumer, "Self-Regulation of Slow Cortical Potentials: A New Treatment for Children with AttentionDeficit/Hyperactivity Disorder,” Pediatrics, Vol. 118, No. 5, 2006, pp. 1530-1540. doi:10.1542/peds.2005-2478

[29] M. Arns, S. de Ridder, U. Strehl, M. Breteler and A. Coenen, "Efficacy of Neurofeedback Treatment in ADHD: The Effects on Inattention, Impulsivity and Hyperactivity: A Meta-Analysis," Clinical EEG and Neuroscience, Vol. 40, No. 3, 2009, pp. 180-189.

[30] J. P. Kline, C. N. Brann and B. R. Loney, “A Cacophony in the Brainwaves: A Critical Appraisal of Neurotherapy for Attention Deficit Disorders," The Scientific Review of Mental Health Practice, Vol. 1, No. 1, 2002, pp. 46-56.

[31] P. M. Ramirez, D. Desantis and L. A. Opler, "EEG Biofeedback Treatment of ADD. A Viable Alternative to Traditional Medical Intervention?" Annals of the New York Academy of Sciences, Vol. 931, 2001, pp. 342-358. doi:10.1111/j.1749-6632.2001.tb05789.x

[32] V. J. Monastra, S. Lynn, M. Linden, J. F. Lubar, J. Gruzelier and T. J. LaVaque, "Electroencephalographic Biofeedback in the Treatment of Attention-Deficit/Hyperactivity Disorder,” Applied Psychophysiology and Biofeedback, Vol. 30, No. 2, 2005, pp. 95-114. doi:10.1007/s10484-005-4305-X

[33] T. J. La Vaque and T. Rossiter, “The Ethical Use of Placebo Controls in Clinical Research: The Declaration of Helsinki,” Applied Psychophysiology and Biofeedback, Vol. 26, No. 1, 2001, pp. 23-37, pp. 61-65. doi:10.1023/A:1009563504319

[34] D. Vernon, A. Frick and J. Gruzelier, "Neurofeedback as a Treatment for ADHD: A Methodological Review with Implications for Future Research," Journal of Neurotherapy, Vol. 8, No. 2, 2004, pp. 53-82. doi:10.1300/J184v08n02 04

[35] T. M. Achenbach, "Manual for the Child Behavior Checklist/4-18 and 1991 Profile,” University of Vermont Department of Psychiatry, Burlington, 1991.

[36] J. M. Swanson, S. B. Wigal, D. Udrea, M. Lerner, D. Agler, D. Flynn, et al., "Evaluation of Individual Subjects in the Analog Classroom Setting: I. Examples of Graphical and Statistical Procedures for within-Subject Ranking of Responses to Different Delivery Patterns of Methylphenidate,” Psychopharmacology Bulletin, Vol. 34, No. 4, 1998, pp. 825-832.

[37] G. J. DuPaul, R. A. Barkley and D. F. Connor, "Stimulants,” In: R. A. Barkley, Ed., Attention-Deficit Hyperactivity Disorder: A Handbook for Diagnosis and Treatment, Guilford Press, New York, 1998, pp. 510-551.

[38] D. Wechsler, "Wechsler Intelligence Scale for Children (WISC-III), San Antonio,” Psychological Corporation, Toronto, 1991.

[39] J. R. Stroop, "Studies of Interference in Serial Verbal Reactions,” Journal of Experimental Psychology, Vol. 18, No. 6, 1935, pp. 643-662. doi:10.1037/h0054651

[40] J. Albaret and M. Migliore, "Manuel du Test de Stroop, ” Editions du Centre de Psychologie Appliquée, Paris, 1999.
[41] C. K. Conners, “Conners' Continuous Performance Test for Windows (CPT II),” Multi-Health Systems Inc., Canada, 2002.

[42] L. Deltour, M. Barathon, V. Quaglino, M. P. Vernier, P. Despretz, M. Boucart, et al., "Children with Benign Epilepsy with Centrotemporal Spikes (BECTS) Show Impaired Attentional Control: Evidence from an Attentional Capture Paradigm,” Epileptic Disorder, Vol. 9, No. 1, 2007, pp. 32-38.

[43] L. Querne and P. Berquin, "Distinct Response Time Distributions in Attention Deficit Hyperactivity Disorder Subtypes," Journal of Attentional Disorders, Vol. 13, No. 1, 2009, pp. 66-77. doi:10.1177/1087054708323006

[44] A. Scheres, J. Oosterlaan, S. J. Wanson, S. Morein-Zamir, N. Meiran, H. Schut et al., "The Effect of Methylphenidate on Three Forms of Response Inhibition in Boys with AD/HD,” Journal of Abnormal Child Psychology, Vol. 31, No. 1, 2003, pp. 105-120. doi:10.1023/A:1021729501230

[45] M. M. Lansbergen, J. L. Kenemans and H. van Engeland, "Stroop Interference and Attention-Deficit/Hyperactivity Disorder: A Review and Meta-Analysis,” Neuropsychology, Vol. 21, No. 2, 2007, pp. 251-262. doi:10.1037/0894-4105.21.2.251

[46] G. J. DuPaul, A. D. Anastopoulos, T. L. Shelton, et al., "Multimethod Assessment of Attention Deficit HyperActivity Disorder: The Diagnostic Utility of Clinic-Based Tests,” Journal of Clinical Child Psychology, Vol. 21, 1992, pp. 394-402. doi:10.1207/s15374424jccp2104_10

[47] H. H. Jasper, “The Ten-Twenty Electrode System of the International Federation,” Electroencephalography and Clinical Neurophysiology, Vol. 10, 1958, pp. 367-380.

[48] J. Cohen, "Statistical Power Analysis for the Behavioral Sciences," 2nd Edition, Lawrence Erlbaum Associates Inc., New Jersey, 1998.

[49] H. S. Leff, D. A. Wieman, B. H. McFarland, J. P. Morrissey, A. Rothbard, D. L. Shern, et al., "Assessment of Medicaid Managed Behavioral Health Care for Persons with Serious Mental Illness,” Psychiatric Services, Vol. 56, No. 10, 2005, pp. 1245-1253. doi:10.1176/appi.ps.56.10.1245

[50] J. L. Rogers, K. I. Howard and J. T. Vessey, “Using Significance Tests to Evaluate Equivalence between Two Experimental Groups,” Psychological Bulletin, Vol. 113, No. 3, 1993, pp. 553-65. doi:10.1037/0033-2909.113.3.553

[51] J. P. Hatch, "Using Statistical Equivalence Testing in Clinical Biofeedback Research,” Biofeedback Self Regulation, Vol. 21, No. 2, 1996, pp. 105-119. doi:10.1007/BF02284690

[52] D. D. Langleben, J. Monterosso, I. Elman, B. Ash, G. Krikorian and G. Austin, "Effect of Methylphenidate on Stroop Color-Word Task Performance in Children with Attention Deficit Hyperactivity Disorder," Psychiatry Research, Vol. 141, No. 3, 2006, pp. 315-320. doi:10.1016/j.psychres.2005.09.007 
[53] A. C. Bedard, A. Ickowicz and R. Tannock, "Methylphenidate Improves Stroop Naming Speed, But Not Response Interference, in Children with Attention Deficit Hyperactivity Disorder,” Journal of Child and Adolescent Psychopharmacology, Vol. 12, No. 4, 2002, pp. 301-309. doi:10.1089/104454602762599844

[54] G. Bush, J. A Frazier, S. L. Rauch, L. J. Seidman, P. J. Whalen, M. A. Jenike, et al., "Anterior Cingulate Cortex Dysfunction in Atten-tion-Deficit/Hyperactivity Disorder Revealed by FMRI and the Counting Stroop,” Biological Psychiatry, Vol. 45, No. 12, 1999, pp. 1542-1552.
doi:10.1016/S0006-3223(99)00083-9

[55] T. Shallice, G. M. Marzocchi, S. Coser, M. Del Savio, R. F. Meuter and R. I. Rumiati, "Executive Function Profile of Children with Attention Deficit Hyper-Activity Disorder," Developmental Neuropsychology, Vol. 21, No. 1, 2002, pp. 43-71. doi:10.1207/S15326942DN2101 3

[56] R. M. Wielkiewicz, "Interpreting Low Scores on the WISC-R Third Factor: It's More than Distractibility. Psychological Assessment,” A Journal of Consulting and Clinical Psychology, Vol. 2, No. 1, 1990, pp. 91-97. 